\title{
Segmentation and visualization of digital in-line holographic microscopy of three-dimensional scenes using reconstructed intensity images
}

\author{
Karen M. Molony ${ }^{a}$, James P. Ryle ${ }^{b-d}$, Susan McDonnell ${ }^{e}$, John T. Sheridan $^{b-d}$ and \\ Thomas J. Naughton ${ }^{a, f}$ \\ ${ }^{a}$ Department of Computer Science, National University of Ireland, Maynooth, Republic of \\ Ireland. \\ ${ }^{b}$ UCD Communications and Optoelectronics Research Centre \\ ${ }^{c}$ SFI Strategic Research Centre in Solar Energy Conversion \\ $d$ School of Electronic, Electrical and Mechanical Engineering \\ College of Engineering, Mathematical and Physical Sciences, University College Dublin, \\ Belfield, Dublin 4, Ireland. \\ $e$ School of Chemical and Bioprocess Engineering, University College Dublin, Belfield, \\ Dublin 4, Ireland

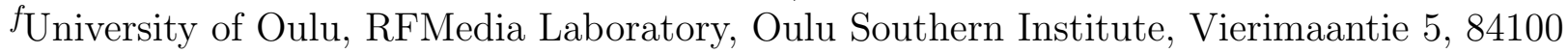 \\ Ylivieska, Finland.
}

\begin{abstract}
This paper demonstrates a technique that could prove useful for extracting three-dimensional (3D) models from a single two-dimensional (2D) digital in-line holographic microscopy (DIHM) recording. Multiple intensity images are reconstructed at a range of depths through a transmissive or partially transmissive scene recorded by DIHM. A two step segmentation of each of these reconstructed intensity images facilitates the construction of a data set of surfaces in 3D. First an adaptive thresholding step and then a border following step are implemented. The surfaces of segmented features are rendered in 3D by applying the marching cubes algorithm to polygonize the data set. Experimental results for a real world DIHM capture of a transmissive glass sample are presented to demonstrate this segmentation and visualization process.
\end{abstract}

\section{INTRODUCTION}

Holography ${ }^{1,2}$ is an imaging science made up of two parts, recording and replay. Traditionally, photographic films were used to record holograms. A hologram encodes three-dimensional (3D) information; intensity and directional information of the optical wave-front. Digital holography is derived from conventional holography but uses a digital area sensor, e.g. a charge coupled device (CCD), instead of an analog photographic medium in order to capture the holograms, and reconstruction is performed digitally on a computer. ${ }^{3,4}$ This has only recently become feasible due to advances in CCD sensors with high spatial resolution and high dynamic range. The in-line microscopic principle originally proposed by Gabor ${ }^{1}$ is the simplest realization of holography and is referred to as digital in-line holographic microscopy (DIHM). ${ }^{5}$ This is the optical recording set-up that we apply in this paper.

Typical microscopy approaches, e.g. confocal microscopy, require dyes to make (quasi-)transparent biological samples, which are compressed between glass slides, visible. DIHM enables biological specimens to be analyzed at a cellular level in a completely unaltered environment in 3D. Objects in the world are 3D, however these objects are imaged in two-dimensions (2D). In order to obtain volumetric data using typical microscopy approaches, physical scanning of a sample is required. DIHM has a significant advantage as 3D information is encoded in a single recording and reconstruction is performed off-line. Therefore relative movement of a sample is not a consideration as no mechanical scanning is required. Manual analysis of this type of data by a biologist is a tedious process.

Applications of Digital Image Processing XXXII, edited by Andrew G. Tescher, Proc. of SPIE Vol. $7443,74431 \mathrm{~F} \cdot$ ? 2009 SPIE - CCC code: 0277-786X/09/\$18 - doi: 10.1117/12.826832 
A difficulty introduced when using DIHM data is that DIHM is an emerging technology with which biologists are not yet familiar. This is compounded by the fact that the DIHM data is in fact 3D data. Extraction of 3D data from DIHM data and the subsequent analysis of the results is currently an unsolved problem in the general case.

It has been shown for a particle field that intensity reconstructions at different depths allows for motion tracking of particles in 3D. ${ }^{5-13}$ Previous 2D segmentation of digital holographic microscopy intensity reconstructions used active contours. ${ }^{14}$ This approach requires an initial predefined geometric shape that evolves dynamically. The process requires random evolution and conditions depicting whether an evolution step is taken. It was shown to be successful for single features in a scene where the initial position of that feature was known. A tomographic approach in off-axis digital holographic microscopy has been shown capable of producing a 3D point cloud of refractive index values of an animal cell that can be visualised using any 3D computer graphics

software. ${ }^{15}$ Extraction of $2.5 \mathrm{D}$ data from macroscopic digital holography scenes has been achieved ${ }^{16}$ by using focus criteria to plot points on a $3 \mathrm{D}$ grid.

In this paper we focus on a long term goal of visualizing 3D features of samples imaged by DIHM. In order to achieve this, reconstructions, at a range of depths, are obtained. The reconstruction depths are equally spaced by a physical distance approximately half of the depth resolution of the system. This ensures that all detectable changes in the axial direction are observed. The range of reconstruction depths were chosen manually for the experiment presented in this paper, however various autofocus techniques are available that can be used to automate this process. ${ }^{17-19}$ We employ a 2D segmentation that is comprised of two parts. The number of features in a scene is irrelevant. An a-priori estimate of feature size is helpful, though not necessary. Adaptive thresholding ${ }^{20}$ is enforced on each intensity reconstruction first and then border following ${ }^{21}$ is applied to each of these thresholded reconstructions. This results in a stack of segmented images corresponding to each reconstruction. This stack is input to the marching cubes algorithm ${ }^{22}$ to polygonize the data set. This renders the surface of the volume that was imaged, and thus a potential 3D segmentation and visualization technique for DIHM is presented.

In Section 2 an overview of the DIHM set-up and reconstruction process is described. The segmentation approach used is described in in Section 3. Section 4 describes the marching cubes algorithm. We present some experimental results in Section 5 and we conclude in Section 6.

\section{DIGITAL IN-LINE HOLOGRAPHIC MICROSCOPY (DIHM)}

Holography is a two-step process. The first step involves the recording of an interference pattern in a plane from an object beam and a reference beam. The second step involves replaying the wavefront of the original object extracted from this recording. Typically the numerical reconstruction is a $2 \mathrm{D}$ description of the wavefront at a specified distance from the camera. The DIHM set-up requires only a point light source, a pinhole, a transparent or partially transparent sample to be imaged, and an intensity recording device. An assumption made when using this set-up is that the object wave is weak with respect to the reference wave, which is an accurate approximation in the case of partially transparent objects e.g. biological samples ${ }^{5}$ or small opaque objects sparsly distributed in the field of view. ${ }^{13}$ This limits applicability of DIHM as described in chapter 9 of. ${ }^{23}$

As shown in Fig. 1, a spherical diverging beam $r(x)$ emerges from a pinhole illuminating an object $f(x)$ a distance $d_{1}$ away. Immediately behind this plane there is an object wave $o(x)=f(x) r(x)$. The interference pattern $H\left(x^{\prime}\right)$ between the propagated reference wave $R\left(x^{\prime}\right)$ and the propagated object wave $O\left(x^{\prime}\right)$ is captured on a CCD a further distance $d_{2}$ away. This captured image then provides the input to the numerical reconstruction part of the imaging system.

Reconstruction of the captured intensity using this set-up is possible digitally on a computer, by numerically calculating a diffraction integral that describes propagation in free space. ${ }^{24}$ The sampling conditions for the Fresnel integral have been formalized ${ }^{25,26}$ and numerical approximations of the Fresnel transform (FST) have been applied successfully for digital hologram reconstruction. ${ }^{26-28}$ Some fast algorithms for calculating free 


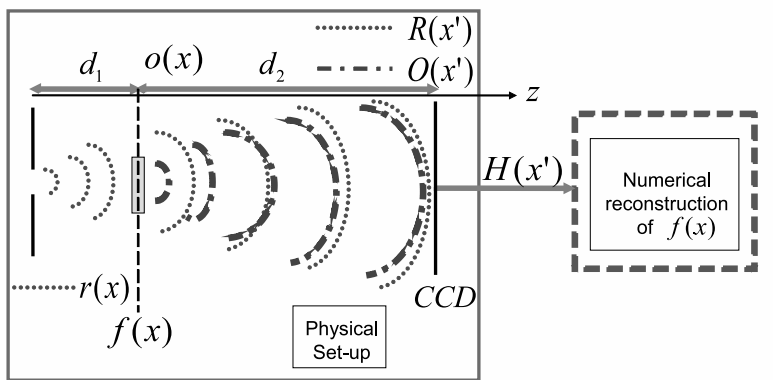

Figure 1. The physical components of a DIHM set-up are a light source, pin hole, sample, CCD and a computer for numerical processing.

space Fresnel diffraction patterns have also been developed ${ }^{29,30}$ and are applied in this paper to reconstruct the DIHM holograms in the experiment described.

\section{SEGMENTATION}

In image processing, segmentation subdivides an image into regions. In digital holography, not all parts of a 3D feature or an imaged 3D object will necessarily be in-focus in a given 2-D reconstruction at a specific depth. At any given depth, a reconstruction will show features in-focus at that depth while all those outof-focus features appear with reduced clarity. ${ }^{31}$ Segmentation can be performed on a single reconstruction at a specific depth, or on multiple independently focused reconstructions at different depths, ${ }^{31}$ which, when combined, can be used to derive a topography of the scene. In conventional digital holography a range of perspectives allow manipulation of the scene to overcome occlusions. ${ }^{32}$ In DIHM such a range is usually not available due to the proximity of the components in the set-up. However, as DIHM is applied to transmissive objects, retrieving segmentations at multiple depths can be achieved without the obstruction of occluding features. For an opaque sample the front plane is all that can be imaged. Reconstruction at a plane beyond it just achieves an out of focus reconstruction of the front plane of the object. For a transmissive sample with some intensity modulation, reconstructing beyond the front plane can be achieved. We show in Section 5 that there are differences between the reconstructions obtained at different depths. Applying an adaptive threshold to filter out-of-focus features in an intensity reconstruction of a DIHM capture allows a successful application of border following defined below.

\subsection{Adaptive Threshold}

In order to remove out-of-focus background features from a reconstructed intensity image a threshold can be applied. Straightforward thresholding is the simplest method of segmentation ${ }^{33}$ whereby pixels with values greater than a given threshold are considered to be in the foreground and all others are assumed to be in the background or vice versa. In DIHM intensity reconstructions we assume that the in-focus features are brighter with respect to the background than out-of-focus features, as we use contrast holograms. ${ }^{5} \mathrm{~A}$ straightforward thresholding operation is not as applicable if the background is not uniform. There is ample opportunity for noise to enhance an uneven background in a DIHM reconstruction, for example the DC term and twin image ${ }^{23}$ speckle noise, ${ }^{34}$ and out-of-focus features themselves can influence the background. Therefore an adaptive threshold ${ }^{20}$ is applicable. As each reconstruction is obtained from the same DIHM capture the recording conditions are unchanged. The same parameters for the adaptive threshold are applied for each reconstruction intensity image. OpenCV ${ }^{35}$ an open source computer vision library, provides an implementation of this which we apply in the grayscale region $\{0-255\}$ on block sizes $\approx 2 \%$ of our image using mean weighting of the pixels. The variable threshold is the weighted average of pixels within a block minus an offset. This provides a binary image of seperated foreground and background features. 


\subsection{Border following}

Border following ${ }^{21}$ is an algorithm that takes a binary image as input and outputs the borders of the objects and holes within those objects in the image. By automatically indexing the 2D raster of the input image, each pixel is checked to see if it is a new foreground pixel connected to background pixel(s). Once such a boundary pixel is identified its connected pixels are examined. Usually the next pixel to be checked is in the same direction that has just been found. This process is repeated for each subsequent connected neighbor pixel, tracing the boundary until no more connected border pixels are found. Typically boundaries of the object are assumed to be in 8-connectivity and boundaries of holes to be in 4-connectivity ${ }^{21}$ and so are treated distinctly in the output. OpenCV provides an implementation using these assumptions. The output is a contour, or a list of points that comprise a curve in an image. ${ }^{35}$ We manipulate this list to only consider contours of a given range of lengths using a-priori knowledge of the sought features in order to limit the number of false feature boundaries identified.

\section{VISUALIZATION}

Segmentation applied to reconstruction intensity images at a range of depths results in a stack of binary contour images. The surface of a volume is constructed from this stack. The data is now represented by slice segmentations through the scene. There are various scanning technologies that obtain data which is stored in a similar manner, e.g. confocal laser scanning microscopy ${ }^{36}$ and magnetic resonance imaging (MRI). ${ }^{37}$ The significant difference with DIHM data is that the slices are all obtained from a single capture and so the volume is not physically scanned. The sample must be transmissive, but not totally transmissive, with some intensity modulation from scattered light so that this can be achieved. Excessive scattering will corrupt reconstructed intensity images. Intensity reconstructions of the DIHM capture at a range of depths spaced by half the depth resolution of the system are the slices. This spacing ensures that we will encounter any axial changes that can be observed by this system. Therefore relative movement of a sample is not a problem. Furthermore no alteration of, or contact with, the sample is required, e.g. in confocal microscopy fluroescence or chemical dyes are required. Some 3D visualization techniques of data stored in this way have been explored. ${ }^{38}$ Marching cubes $^{22}$ is an algorithm that was developed specifically for visualizing this type of data and has proved effective for the polygonization of similar scalar data. ${ }^{39}$

\subsection{Marching Cubes}

Marching cubes ${ }^{22}$ is an algorithm that generates triangles to represent surfaces. A 3D raster is subdivided into cubes. Each vertex of the cube is numbered $0-7$ as shown in Fig. 2.

Based on the values of the vertices of the cube with respect to an isosurface value, that cube is considered to be wholly inside or outside of the surface, or intersected by the surface. If some data values at vertices are higher than the isosurface value and some are lower then triangles are drawn on the edges of the cube to indicate the intersections. There are $2^{8}$ possibilities and so a known look up table is used which returns a twelve bit result, one for each edge of the cube, to show intersections on this cube. A look up table of corresponding triangles is also available for computational efficiency. An example is shown in Fig. 3 where vertex 3 and 5 are below the isosurface and the resulting intersecting triangles are drawn.

\section{EXPERIMENTAL RESULTS}

In this paper we detail the steps involved in the segmentation and visualization of transmissive or partially transmissive 3D scenes. We demonstrate these steps using a scene of a broken $1 \mathrm{~mm}$ thick glass slide captured using the DIHM set-up shown in Fig. 1. The sample is totally transmissive though the edge scatters some light so is visible in the reconstructed intensity images. We segment and visualize this edge in 3D.

The following results were captured using a DIHM set-up with a $2 \mu \mathrm{m}$ diameter pinhole illuminated by a light source with $\lambda=632 \mathrm{~nm}$. The sample is placed $14 \mathrm{~mm}$ from the pinhole $\left(d_{1}\right)$. A CCD sensor, with $1000 \times$ 1000 pixels of width and height $8 \mu \mathrm{m}(d x)$, is placed $50 \mathrm{~mm}$ from the pinhole $\left(d_{1}+d_{2}\right)$. The magnification of 


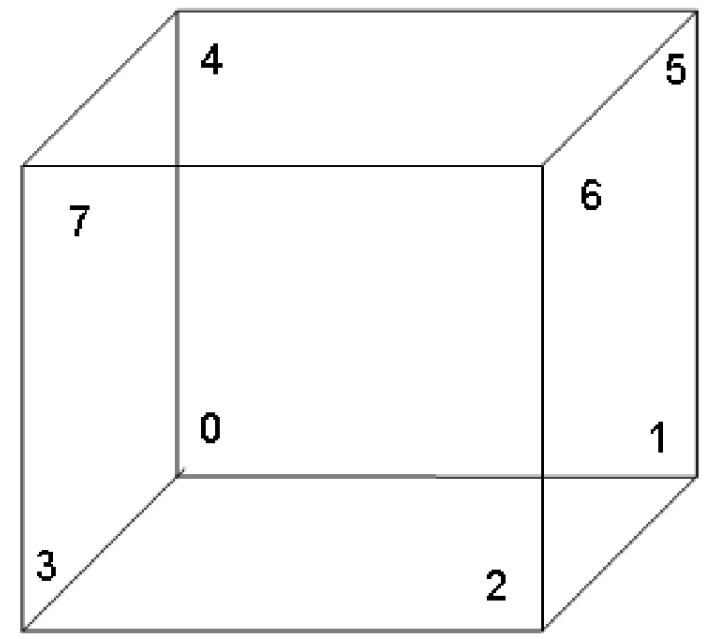

Figure 2. A volume is subdivided into cubes. The vertices are numbered $0-7$.

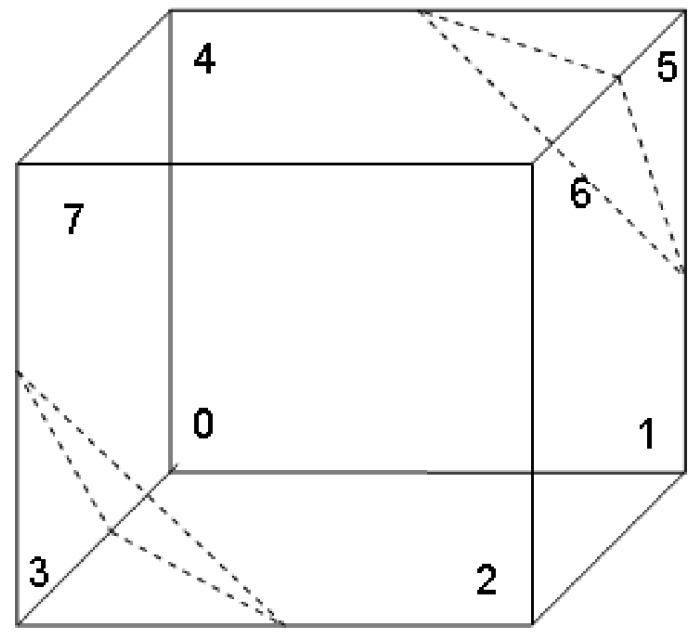

Figure 3. Triangles drawn for a cube where only vertex 3 and vertex 5 are below the isosurface.

the system is thus 3.57 , i.e. $M=\left[\left(d_{1}+d_{2}\right) / d_{1}\right]$. The depth resolution $\approx 110 \mu \mathrm{m}$, i.e. $[d z=d x / N A$, where the numerical aperture is $N A=(W / 2) / \sqrt{(W / 2)^{2}+\left(d_{1}+d_{2}\right)^{2}}$, and $W$ is the width of the $\left.\mathrm{CCD}^{5}\right]$.

A contrast hologram ${ }^{40}$ such as that given in the example shown in Fig. 4, is a DIHM recording of the sample minus a recording of free space. It was determined manually that the range of depths from the front of the slide to the back of the slide were in the range of: $36-37 \mathrm{~mm}$ from the pinhole. For reconstruction of DIHM recordings using the angular spectrum method ${ }^{29}$ a distance $d=\left[d_{2}\left(d_{1}+d_{2}\right)\right] / d_{1}$ is typically used. ${ }^{41}$ For each reconstruction some distance from the hologram plane, the distance from the reconstruction plane to the pinhole is different. Therefore the ratio of $d_{1}$ to $d_{2}$ is altered and so the reconstruction distances corresponding to the equally spaced physical distances are not equally spaced and range from $0.1218 \mathrm{~m}$ to $0.1359 \mathrm{~m}$. To account for this, nineteen reconstructions from planes seperated by $55 \mu \mathrm{m}$ are obtained and encompass the $1 \mathrm{~mm}$ thick glass slide. Four examples of reconstructions at different distances within this range are presented in Fig. 5. In Fig. 5. (A,C, and D) in-focus features on the edge of the imaged glass slide are identified. These features are not in focus in the other planes. An enlargement of these regions is shown in Fig. 6 .

Features in these scenes extend beyond the viewing space, thus an artificial border (of ten pixels with the 


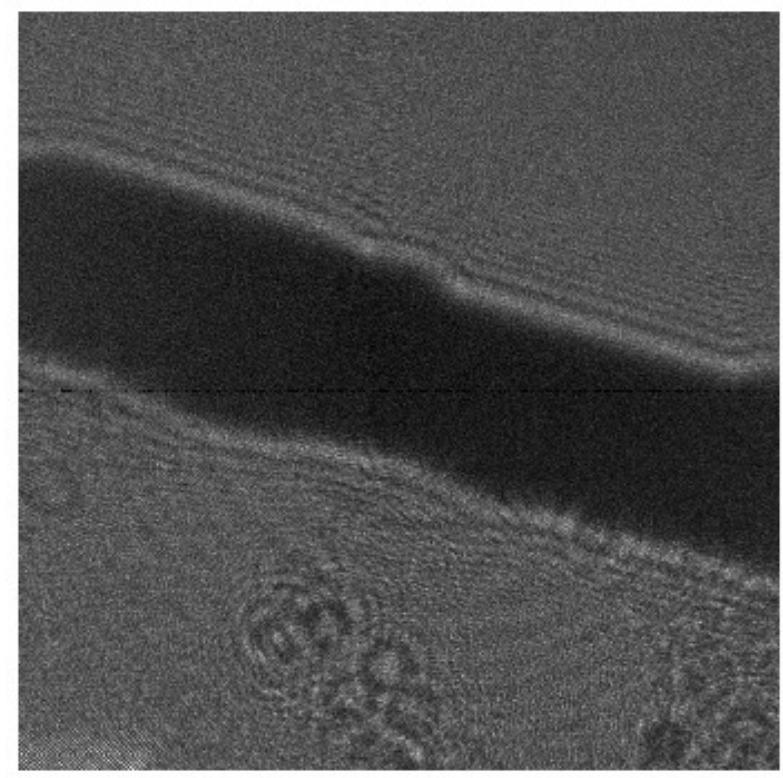

Figure 4. Contrast hologram of the edge of a broken glass slide of $1 \mathrm{~mm}$ thickness obtained using the current DIHM set-up. The upper right section is free space, the middle section is the broken edge of the shard, and the bottom left secion is the body of the glass slide. Pinhole width $=2 \mu \mathrm{m}, d_{1}=14 \mathrm{~mm}, d_{2}=50 \mathrm{~mm}, \mathrm{NA}=0.0725$ and $\lambda=632 \mathrm{~nm}$.
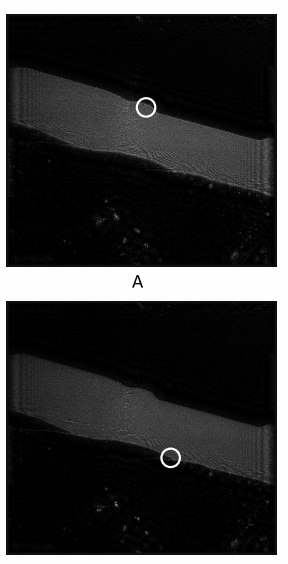

C
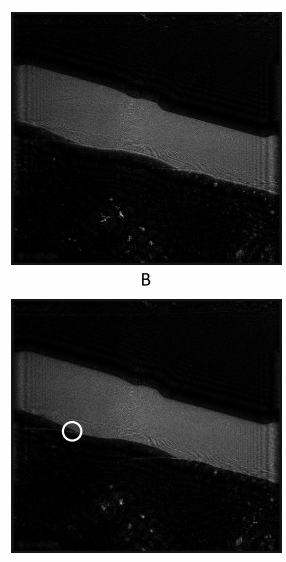

D

Figure 5. Four reconstruction intensity images within the focus range seperated by a physical distance of $330 \mu \mathrm{m}$. White circles indicate a feature that is in focus at that plane. These regions are enlarged in Fig. 6. (A) Intensity reconstruction at the depth of the front plane, $36 \mathrm{~mm}$ from the camera. (B) Intensity reconstruction at a depth $330 \mu \mathrm{m}$ from the front plane. (C) Intensity reconstruction at a depth $660 \mu \mathrm{m}$ from the front plane. Finally (D) Intensity reconstruction at the depth of the back plane, $37 \mathrm{~mm}$ from the camera.

mean value of the intensity reconstruction) is added. The sought feature in this scene, the edge of the shard of glass, does not have a consistent intensity and so an averaging filter is applied in a $9 \times 9$ block. This smoothes the image resulting in a more consistent foreground. An adaptive threshold using an offset of -10 , to segment all pixels $\approx 4 \%$ brighter than the mean pixel brightness in a block, is applied in non-overlapping blocks of $331 \times 331$. The block size is chosen such that its area is greater than the thickest portion of foreground. Results of adaptive thresholding applied to the four reconstructions in Fig. 5 are shown in Fig. 7. Border following is then used to obtain contours of length $\geq 800$ pixels as shown in Fig. 8. This ensures that only those features that are greater than the expected minimum size of the feature are detected. The remaining identified borders are considered contours. The contours for each reconstructed plane comprise the data set of surfaces in 3D. This is input to the marching cubes algorithm which renders the surface of the feature in 


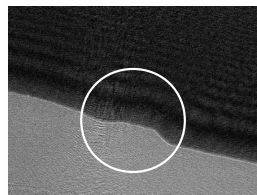

i

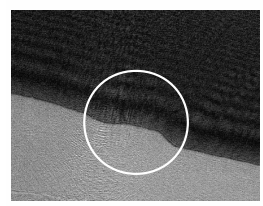

iv

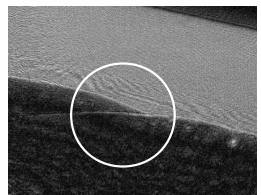

ii

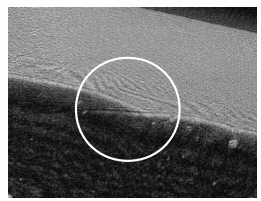

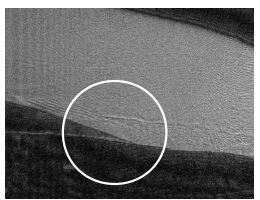

iii

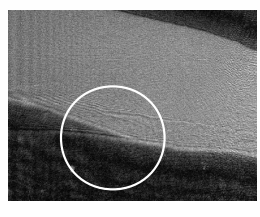

Figure 6. (i-iii) are a closer view of the in-focus features shown in Fig. 5 (A,C and D). (iv-vi) are a closer view of the same regions corresponding to (i-iii) in Fig. 5.B. It can be observed, in particular for the latter two cases shown, that the observed in-focus feature in (i-iii) is subtly less focused in (iv-vi)

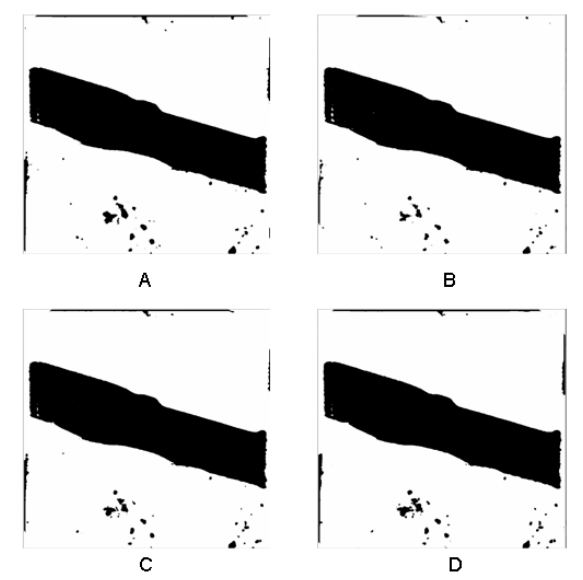

Figure 7. (A-D) show the result of adaptive thresholding the intensity reconstructions shown in Fig. 5 (A-D) respectively.

3D. Different perspectives of the rendered scene are shown in Fig. 9.

This technique is applicable to any transmissive or partially transmissive scene captured using a digital holographic microscopy set-up. Biological samples are (quasi-)transparent and suitable for 3D visualization in this way assuming a depth resolution less than at most a third of the diameter of a cell so that at least three distinct planes can be reconstructed. Typically at least three depths are required to construct a volume. In order to achieve a depth resolution so that biological cells can be imaged, e.g. a red blood cell has $\approx 30 \mu \mathrm{m}$ diameter, a higher numerical aperture is required which may be achieved by using a smaller pinhole or a larger area recording device.

\section{CONCLUSIONS}

We have segmented multiple 2D reconstructions of a scene at different depths from a DIHM single hologram of a transmissive scene with some intensity modulation. These $2 \mathrm{D}$ segmentations were used to model the surface of the imaged sample in 3D. This was achieved by first applying an adaptive threshold to numerical reconstructions for a range of depths of a single DIHM hologram. The resulting binary images for each plane were input to a border following algorithm yielding a list of contours for each plane. Only contours within a specified range of pixel lengths were selected and so a contour image for each plane of the in-focus features at the corresponding reconstruction depth was obtained. Finally the marching cubes algorithm was applied 


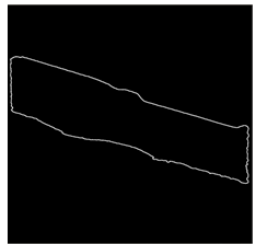

A

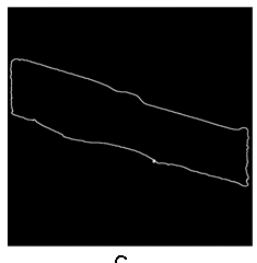

C

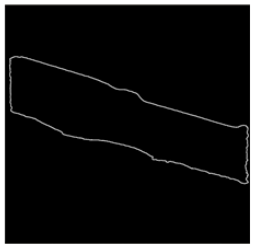

B

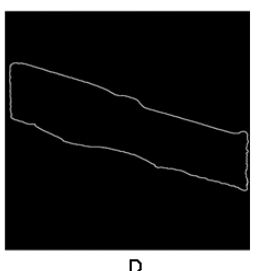

D

Figure 8. (A-D) show the result of border following applied to the thresholded reconstruction intensity images shown in Fig. 7 (A-D) respectively.
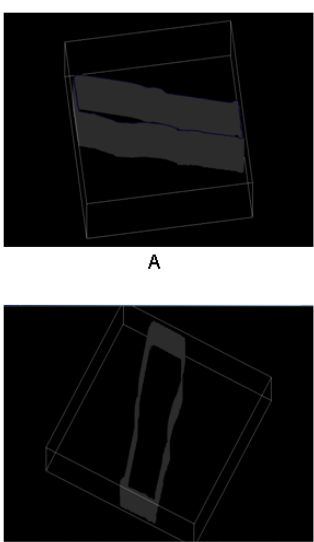

C

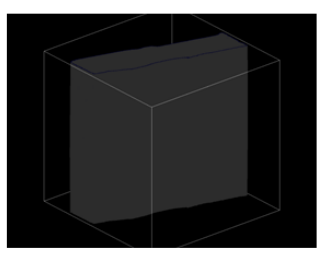

B

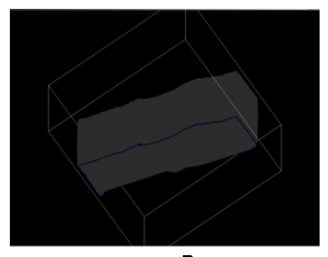

D

Figure 9. The rendering of the edge of the glass slide in 3D that is achieved by applying the marching cubes algorithms to the stack of contour slices can be rotated. The rendering has been placed in a white wire cube box so that orientation can be perceived. (A-D) show four rotations of the $3 \mathrm{D}$ rendering.

to these contour images to polygonize the surface of the segmented feature. This allowed a 3D visualization of the surface of the imaged feature. The steps involved in this process were demonstrated using a test case of a real world hologram of a broken shard of glass. Future work on this topic requires DIHM recording of volumes of transmissive samples, e.g. biological samples, with more obvious feature focus differences between reconstructed planes.

\section{ACKNOWLEDGEMENTS}

We thank the Irish Research Council for Science, Engineering and Technology, Enterprise Ireland and Science

Foundation Ireland, under the National Development Plan for funding this research. The authors wish to thank SPIE for supporting conference attendance through the Student Chapter Travelling Officer Grant programme.

\section{REFERENCES}

11] Gabor, D., "A new microscopic principle," Nature 161, 777-778 (1948).

[2] Leith, E. N. and Upatnieks, J., "Wavefront reconstruction with diffused illumination and threedimensional objects." J. Opt. Soc. Am. 54, 1295-1301 (1964).

[3] Kreis, T. M., [Handbook of Holographic Interferometry], Wiley-VCH (2005). 
[4] Schnars, U. and Jüptner, W. P. O., [Digital Holography], Springer (2004).

[5] Garcia-Sucerquia, J., Xu, W., Jericho, S. K., Klages, P., Jericho, M. H., and Kreuzer, H. J., "Digital in-line holographic microscopy," Appl. Opt. 45, 836-850 (2006).

[6] Adams, M., Kreis, T. M., and Jüptner, W. P. O., "Particle size and position measurement with digital holography," Proc. SPIE 3098 , 234-240 (1997).

[7] Buraga Lefebvre, C., Coëtmellec, S., Lebrun, D., and Özkul, C., "Application of wavelet transform to hologram analysis: three-dimensional location of particles," Opt. and Lasers in Eng. 33, 409-421 (2000).

[8] Xu, L., Peng, X., Miao, J., and Asundi, A. K., "Studies of digital microscopic holography with applications to microstructure testing," Appl. Opt. 40, 5046-5051 (2001).

[9] Xu, W., Jericho, M. H., Kreuzer, H. J., and Meinertzhagen, I. A., "Tracking particles in four dimensions with in-line holographic microscopy," Opt. Lett. 28, 164-166 (2003).

[10] Dubois, F., Callens, N., Yourassowsdy, C., Hoyos, M., Kurowski, P., and Monnom, O., "Digital holographic microscopy with reduced spatial coherence for three-dimensional particle flow analysis," Appl. Opt. 45, 846-870 (2006).

[11] Soulez, F., Denis, L., Fournier, C., Thiébaut, É., and Goepfert, C., "Inverse-problem approach for particle digital holography: accurate location based on local optimization," J. Opt. Soc. Am. A 24, 1164-1171 (2007).

[12] Sun, H., Song, B., Dong, H., Reid, B., Player, M. A., Watson, J., and Zhao, M., "Visualization of fast-moving cells in vivo using digital holographic video microscopy," J. Biomed. Opt. 13, 014007 (2008).

[13] Kempkes, M., Darakis, E., Khanam, T., Rajendran, A., Kariwala, V., Mazzotti, M., Naughton, T. J., and Asundi, A. K., "Three dimensional digital holographic profiling of micro-fibers," Opt. Express 17, 2938-2943 (2009).

[14] DaneshPanah, M. and Javidi, B., "Segmentation of 3D holographic images using bivariate jointly distributed region snake," Opt. Express 14, 5143-5153 (2006).

[15] Charrière, F., Pavillon, N., Colomb, T., Depeursinge, C., Heger, T., Mitchell, E., Marquet, P., , and Rappaz, B., "Living specimen tomography by digital holographic microscopy: morphometry of testate amoeba," Opt. Express 14, 7005-7013 (2006).

[16] Ma, L., H.Wang, Li, Y., and Jin, H., "Numerical reconstruction of digital holograms for three-dimensional shape measurement," J. Opt. A: Pure and Appl Opt. 6, 396-400 (2004).

[17] Ferraro, P., Coppola, G., Nicola, S. D., Finizio, A., and Pierattini, G., "Digital holographic microscope with automatic focus tracking by detecting sample displacement in real time," Opt. Lett. 28, 1257-1259 (2003).

[18] Liebling, M. and Unser, M., "Autofocus for digital fresnel holograms by use of a fresnelet-sparsity criterion," J. Opt. Soc. Am. A 21, 2424-2430 (2004).

[19] Dubois, F., Schockaert, C., Callens, N., and Yourassowsky, C., "Focus plane detection criteria in digital holography microscopy by amplitude analysis," Opt. Express 14, 5895-5908 (2006).

[20] Chan, F. H. Y., Lam, F. K., and Zhu, H., "Adaptive thresholding by variational method," IEEE Trans. Imag. Proc. 7, 468-473 (1998).

[21] Suzuki, S. and Be, K., "Topological structural analysis of digitized binary images by border following," Comp. Vis., Graph., and Imag. Proc. 30, 32-46 (1985).

[22] Lorensen, W. E. and Cline, H. W., "Marching cubes a high resolution 3D surface construction algorithm," Comp. Graph. 21, 163-169 (1987).

[23. Goodman, J. W., [Introduction to Fourier Optics], Roberts \& Company Publishers (2004).

24. Pedrini, G., Frning, P., Fessler, H., and Tiziani, H. J., "Inline digital holographic interferometry," Appl. Opt. 37, 6262-6269 (1998).

[25. Gori, F., "Fresnel transform and sampling theorem," Opt. Eng. 39, 293-297 (1981).

[26] Stern, A. and Javidi, B., "Analysis of practical sampling and reconstruction from fresnel fields," Opt. Eng. 43, 239-250 (2004).

[27] Zhang, Y., Pedrini, G., Osten, W., and Tiziani, H. J., "Reconstruction of in-line digital holograms from two intensity measurements," Opt. Lett. 29, 1287-1789 (2004).

[28] Frauel, Y., Naughton, T. J., Matoba, O., Tajahuerce, E., and Javidi, B., "Three-dimensional imaging and processing using computational holographic imaging," Proc. IEEE 94, 636-653 (2006).

[29] Mas, D., Garcia, J., Ferreira, C., Bernardo, L. M., and Marinho, F., "Fast algorithms for free-space diffraction patters calculation," Opt. Commun. 164, 233-245 (1999).

[30] Mas, D., Prez, J., Hernndez, C., Vzquez, C., Miret, J., and Illueca, C., "Fast algorithms for free-space diffraction patters calculation," Opt. Commun. 227, 245-258 (2003).

[31] McElhinney, C. P., McDonald, J. B., Castro, A., Frauel, Y., Javidi, B., and Naughton, T. J., "Depthindependent segmentation of macroscopic three-dimensional objects encoded in single perspectives of digital holograms," Opt. Lett. 32, 1229-1231 (2007). 
[32] Maycock, J., McElhinney, C. P., Hennelly, B. M., Naughton, T. J., Donald, J. B. M., and Javidi, B., "Reconstruction of partially occluded objects encoded in three-dimensional scenes using digital holograms," App. Opt. 45, 2975-2985 (2006).

[33] Sonka, M., Hlavac, V., and Boyle, R., [Image Processing, Analysis, and Machine Vision], Brooks/Cole Publishing Company, 2 ed. (1999).

[34] Maycock, J., Hennelly, B. M., McDonald, J. B., Frauel, Y., Castro, A., Javidi, B., and Naughton, T. J., "Reduction of speckle in digital holography by discrete fourier filtering," J. Opt. Soc. Am. A 24(6), 1617-1622 (2007).

[35] Bradski, G. and Kaehler, A., [Learning OpenCV Computer Vision with the OpenCV Library], O'Reilly Media, Inc (2008).

[36] Carlsson, K., Danielsson, P. E., Lenz, R., Liljeborg, A., Majlbf, L., and Aslund, N., "Three-dimensional microscopy using a confocal laser scanning microscope," Opt. Lett. 10, 53-55 (1985).

[37] Lauterbur, P. C., "Image formation by induced local interactions: Examples of employing nuclear magnetic resonance," Nature 242, 190-191 (1973).

[38] O'Conor, K., Voorheis, H. P., and O'Sullivan, C., "3D visualisation of confocal fluorescence microscopy data," Eurographics Ireland Workshop , 49-54 (2004).

[39] Tiede, U., Hoene, K. H., Bomans, M., Pommert, A., Riemer, M., and Wiebecke, G., "Investigation of medical 3D-rendering algorithms," Comp. Graph. and Appl., IEEE 10, 41-52 (1990).

[40] Barton, J. J., "Photoelectron holography," Phys. Rev. Lett. 61, 1356-1359 (1988).

[41] Molony, K. M., Hennelly, B. M., Kelly, D. P., and Naughton, T. J., "Reconstruction algorithms applied to in-line gabor digital holographic microscopy." in preparation 2009. 